\title{
An appraisal of rehabilitation regimes used for improving functional outcome after total hip replacement surgery
}

\author{
Tosan Okoro ${ }^{1,2,3^{*}}$, Andrew B Lemmey ${ }^{2}$, Peter Maddison ${ }^{2}$ and John G Andrew ${ }^{3}$
}

\begin{abstract}
This study aimed to systematically review the literature with regards to studies of rehabilitation programmes that have tried to improve function after total hip replacement (THR) surgery. 15 randomised controlled trials were identified of which 11 were centre-based, 2 were home based and 2 were trials comparing home and centre based interventions. The use of a progressive resistance training (PRT) programme led to significant improvement in muscle strength and function if the intervention was carried out early $(<1$ month following surgery) in a centre (6/11 centre-based studies used PRT), or late ( $>1$ month following surgery) in a home based setting (2/2 home based studies used PRT). In direct comparison, there was no difference in functional measures between home and centre based programmes (2 studies), with PRT not included in the regimes prescribed. A limitation of the majority of these intervention studies was the short period of follow up. Centre based program delivery is expensive as high costs are associated with supervision, facility provision, and transport of patients. Early interventions are important to counteract the deficit in muscle strength in the affected limb, as well as persistent atrophy that exists around the affected hip at 2 years post-operatively. Studies of early home-based regimes featuring PRT with long term follow up are needed to address the problems currently associated with rehabilitation following THR.
\end{abstract}

Keywords: Total hip replacement, Hip arthroplasty, Resistance training, Rehabilitation, Functional outcome, Muscle wasting

\section{Background}

Symptomatic hip osteoarthritis occurs in $3 \%$ of the elderly [1] and is associated with poor general health status [2]. Treatment strategies for hip pain have usually involved conservative measures (analgesia, exercise, education, weight reduction), with surgical intervention (total hip replacement; THR) being the most effective treatment for end stage disease [3]. The National Joint Registry for England and Wales reports that the number of primary total hip replacements performed in England and Wales in 2009/2010 totaled 79413, which is a steady rise from the numbers reported in 2008/2009 (77608) and 2006/2007 (51981)[4].

The most common preoperative complaints by patients who elect to have THR are pain and loss of

\footnotetext{
* Correspondence: tosanwumi@hotmail.com

${ }^{1}$ School of Medical Sciences (SMS)/School of Sport Health and Exercise Science (SSHES), Bangor University, Penrallt Road, Bangor LL57 2AS, UK Full list of author information is available at the end of the article
}

mobility $[1,5]$. It therefore follows that the most commonly reported outcomes of THR in the literature relate to pain relief and restoration of mobility [1]. It is clear that a major predictor of outcome after THR is the preoperative status - worse preoperative status is followed by a poorer absolute outcome as defined by several outcome measures [6]. Outcome studies of pain reduction and range of motion restoration, usually conducted 3 to 6 months after THR, indicate an overall satisfaction by patients and physicians [7]. However, outcome studies performed at least 1 year after THR reveal that limitations in physical function remain even in the absence of pain. These persisting impairments include decrements of $10-21 \%$ in muscle strength and postural stability of the involved hip relative to the non-operated hip at 1 year post-THR surgery $[8,9]$, with these deficits still evident 2 years after surgery $[2,10]$. Interestingly, Long et al. [10] reported that muscle weakness during stance, along with deterioration of the Harris Hip score, were

\section{C) Biomed Central}


the most consistent findings in patients who developed loosening of the hip components; implying that muscle weakness may result in reduced protection of the prosthetic implant fixation.

Prior to surgery, there is a general deficit in muscular strength along the affected limb as compared to the contra-lateral (healthy) side in patients with unilateral hip osteoarthritis (OA) [11], and muscles such as the abductors, vastii, rectus femoris and psoas show marked atrophy. This is evidenced by reduced cross sectional area and an infiltration of non-contractile components; i.e. an average $10 \%$ increase in fatty infiltration of muscle (myosteatosis) in the affected limb compared to the healthy one [11]. As well as reducing muscle strength, myosteatosis also exacerbates fall risk [12]. This muscular dysfunction is likely to contribute to the reduced ambulatory capacity of OA patients, as loss of lowerlimb muscle strength has been shown to predict the onset of activities of daily living dependence in the elderly [13]. Consistent with the persisting functional deficits following surgery, these atrophic changes about the hip are still evident up to 2 years following THR surgery [11]. Frail elderly persons with sarcopenia often undergo musculoskeletal-related surgery such as THR, and the hospitalisation-associated immobilisation further compromises the skeletal system, with potentially grave consequences [14]. Earlier operation may prevent the development of persistent atrophic changes that occur after THR and there is a suggestion by Rasch et al. [11] that fatty infiltration may be reversed by intensive rehabilitation [11].

There have been considerable technical efforts towards optimising surgical treatment of patients with arthritis of the hip, for example with over 100 varieties of hip prostheses being available, multiple types of bearing couples and several surgical approaches. As technology and surgical techniques for total hip replacement (THR) improve, patient expectations, including for an early return to normal physical function and activities, have also increased [15]. However, the actual health gain for many of these innovations relative to "standard" THR is small in terms of patient function and quality of life [16].

In the past, a prolonged hospital stay after THR surgery incorporated a period of supervised rehabilitation to try to achieve restoration of physical function. However, due to the introduction of initiatives such as integrated care pathways and considerations of cost and increasing patient satisfaction, the length of hospital stay following joint replacement has been substantially reduced [17]. Mean length of stay after THR over the past decade has declined from 3 weeks to 4 days [18]. Rehabilitation is therefore increasingly important following total hip replacement. The aim of this review is to systematically investigate the literature with regards to the highest-level evidence (randomised controlled trials) for studies of rehabilitation programmes that have tried to improve function after this common surgical intervention.

\section{Methods}

Studies were eligible for the review if they met the following criteria: 1) randomised controlled trial of exercise rehabilitation interventions to improve functional outcome in the post-operative period; 2) target population includes patients undergoing total hip arthroplasty for osteoarthritis; and 3) publication in English language. For the purpose of this review, early interventions occurred $\leq 1$ month after surgery and late interventions were $\geq 1$ month after surgery. This distinction is important as it has been noted that muscle strength declines $4 \%$ per day during the first week of immobilisation after major surgery, making it important that rehabilitation is commenced as soon as possible afterwards [19].

Studies were identified from computerized search of MEDLINE (1950 to date), EMBASE (1980 to date), and CINAHL databases. A set was created using the terms: 'total hip arthroplasty AND replacement' and yielded 6559 articles. A search strategy was then built by adding the terms 'exercise', 'rehabilitation', 'physiotherapy', 'functional outcome' to 'total hip arthroplasty AND replacement'. Restriction of the articles obtained from the computerised search to randomised controlled trials and ensuring that the intervention was timed in the post-operative period led to the identification of 15 appropriate studies (Table 1). The studies identified were assessed using the following parameters: 1) whether they were home or centre based, 2) the follow up period used for functional assessment, 3) the interval from surgery to the rehabilitation intervention, 4) the exercise intervention carried out, 5) the outcome measures utilised, and 6) any evidence of dislocation as a complication. For the last parameter listed (dislocation), contact by email was made with the author of any study in which the rate of dislocation was not documented in the article.

\section{Results}

From Table 1, it can be seen that 11 interventions were performed in a rehabilitation centre, 2 involved a direct comparison between home and centre based interventions and 2 trials were home based. The data shows that, if early intervention is defined as commencing within a month of surgery, such an intervention is more likely to have a beneficial effect if it is performed in a centre and involves progressive resistance training (PRT; i.e. strength training wherein the resistance (weight) lifted is increased in accordance with improved strength 
Table 1 Characteristics of randomised controlled trials on hip arthroplasty rehabilitation interventions to improve functional outcome

\begin{tabular}{|c|c|c|c|c|c|c|c|c|}
\hline Article & $\begin{array}{l}\text { Number of } \\
\text { participants }\end{array}$ & Site & $\begin{array}{l}\text { Follow } \\
\text { up } \\
\text { period }\end{array}$ & $\begin{array}{l}\text { Interval from } \\
\text { surgery to } \\
\text { intervention }\end{array}$ & Exercise intervention & Outcome measures & $\begin{array}{l}\text { Dislocation } \\
\text { rate }\end{array}$ & Limitations \\
\hline $\begin{array}{l}\text { Galea et al., } \\
2008 \text { [20] }\end{array}$ & $\begin{array}{l}\text { Home based } \\
\text { group }(\mathrm{n}= \\
\text { 12); } \\
\text { Centre based } \\
\text { group ( } \mathrm{n}= \\
11)\end{array}$ & $\begin{array}{l}\text { Centre } \\
\text { and } \\
\text { Home }\end{array}$ & $\begin{array}{l}8 \\
\text { weeks }\end{array}$ & $\begin{array}{l}\text { Immediate } \\
\text { post-operative } \\
\text { period }\end{array}$ & $\begin{array}{l}\text { All participants: Standard inpatient } \\
\text { physiotherapy with functional tasks, } \\
\text { instructions to take home and } 4 \\
\text { visits at home by physiotherapist } \\
\text { Home group: Exercise as above with } \\
\text { no advice or further instruction } \\
\text { Centre group: } 2 \text { visits/week for } 45 \\
\text { minutes each time. } 5 \text { bouts of } \\
\text { exercise per week }\end{array}$ & $\begin{array}{l}\text { No differences in both groups at } \\
\text { final follow up although all } \\
\text { parameters improved significantly } \\
\text { from baseline in both groups. } \\
\text { Timed up and go: centre } 11.1 \pm 2.5 \\
\text { s vs home } 11.7 \pm 1.5 \text {. } \\
6 \text { minute walking test: centre } 427 \pm \\
78.2 \mathrm{~m} \text { vs home } 457.8 \pm 112.2 \mathrm{~m} \text {. } \\
\text { Stair Climb: centre } 3.1 \pm 1.8 \mathrm{~s} \text { vs. } 2.9 \\
\pm 0.5 \mathrm{~s} \text {. }\end{array}$ & None recorded & $\begin{array}{l}\text { Patients had significant access to } \\
\text { advice and physiotherapy visits. Even } \\
\text { though they had the instructions } \\
\text { and no advice in the home group, } \\
\text { as part of standard protocol, they } \\
\text { could all see physiotherapists on a } \\
\text { further } 3 \text { or } 4 \text { occasions if they } \\
\text { requested it. }\end{array}$ \\
\hline $\begin{array}{l}\text { Giaquinto } \\
\text { et al., } 2010 \\
\text { [21] }\end{array}$ & $\begin{array}{l}\text { Control }(n= \\
33) \text {, } \\
\text { Intervention } \\
(n=31)\end{array}$ & Centre & $\begin{array}{l}6 \\
\text { months }\end{array}$ & $<10$ days & $\begin{array}{l}\text { Control group: Physiotherapy }+ \\
\text { 'neutral' massage of scar } \\
\text { Intervention group: Hydrotherapy in } \\
\text { special pool for } 40 \text { minutes after } 20 \\
\text { minutes of passive joint exercises } \\
\text { All sessions performed } \times 6 / \text { week for } 3 \\
\text { weeks }\end{array}$ & $\begin{array}{l}\text { At } 6 \text { months: } \\
\text { WOMAC pain subscale: } \\
\text { No pain } 45.6 \% \text { intervention group vs. } \\
23 \% \text { control } \\
\text { WOMAC stiffness subscale: } \\
\text { No stiffness } 67.7 \% \text { intervention } \\
\text { group vs. } 35.8 \% \text { control } \\
\text { WOMAC function subscale: } \\
\text { Score of } 0 \text { in function } 19.3 \% \\
\text { intervention group vs. } 2.56 \% \text { control }\end{array}$ & None recorded & $\begin{array}{l}\text {-3 week follow-up data initially } \\
\text { reported by authors showed } \\
\text { objective improvements in speed; } \\
\text { stance for example but no further } \\
\text { attempt was made to see if this was } \\
\text { maintained at } 6 \text { months. } \\
\text {-No absolute values of the WOMAC } \\
\text { subscales given? effect sizes } \\
\text { between groups }\end{array}$ \\
\hline $\begin{array}{l}\text { Greameaux } \\
\text { et al., } 2008 \\
\text { [22] }\end{array}$ & $\begin{array}{l}\text { Intervention } \\
\mathrm{n}=16 ; \\
\text { Control } \mathrm{n}= \\
16\end{array}$ & Centre & 45 days & $\begin{array}{l}\text { Immediate } \\
\text { post-operative } \\
\text { period }\end{array}$ & $\begin{array}{l}\text { Intervention group: low frequency } \\
\text { electrical stimulation of both } \\
\text { quadriceps and calf muscles } \\
\text { bilaterally. } 1 \text { hour session } 5 \text { days a } \\
\text { week for } 5 \text { weeks and conventional } \\
\text { physiotherapy ( } 2 \text { hours a day for } 5 \\
\text { days/week for } 5 \text { weeks ( } 25 \text { sessions)) } \\
\text { Control group: Conventional } \\
\text { physiotherapy - range of motion } \\
\text { exercises, muscle strengthening } \\
\text { static and dynamic }\end{array}$ & $\begin{array}{l}\text { Maximal isometric knee extension: } \\
\text { Significant } \uparrow \text { in power of operated } \\
\text { limb for intervention vs control ( } 66.7 \\
\mathrm{~N}(77 \%) \text { vs } 26.7 \mathrm{~N}(23 \%)), p<0.05 \text {. } \\
\text { No significant difference for length } \\
\text { of stay nor walking assessment } \\
\text { (6MWT and } 200 \mathrm{mFWT} \text { ) }\end{array}$ & None recorded & $\begin{array}{l}\text {-Small sample size } \\
\text {-Absence of a true placebo group } \\
\text {-Absence of standardisation for the } \\
\text { rehabilitation programme }\end{array}$ \\
\hline Article & $\begin{array}{l}\text { Number of } \\
\text { participants }\end{array}$ & Site & $\begin{array}{l}\text { Follow } \\
\text { up } \\
\text { period }\end{array}$ & $\begin{array}{l}\text { Interval from } \\
\text { surgery to } \\
\text { intervention }\end{array}$ & Exercise intervention & Outcome measures & $\begin{array}{l}\text { Dislocation } \\
\text { rate }\end{array}$ & Limitations \\
\hline $\begin{array}{l}\text { Hesse et al., } \\
2003 \text { [23] }\end{array}$ & $\begin{array}{l}\text { Control } n= \\
40 \\
\text { Intervention } \\
n=40\end{array}$ & Centre & $\begin{array}{l}12 \\
\text { months }\end{array}$ & $\begin{array}{l}\text { Within } 3 \\
\text { weeks post- } \\
\text { operatively }\end{array}$ & $\begin{array}{l}\text { All patients: } 45 \text { minute individualised } \\
\text { treatment on each of } 10 \text { consecutive } \\
\text { days including passive physiotherapy } \\
\text { (massage, heat ultrasound), group } \\
\text { therapy in pool. } \\
\text { Control: Passive hip and knee } \\
\text { mobilisation, strengthening of hip } \\
\text { abductor and extensor muscles, gait } \\
\text { retraining on floor and stairs } \\
\text { Intervention: Treadmill training after } \\
\text { above hip and knee mobilisation( } 20 \\
\text { min days 1-5); days 6-10, } 35 \text { minutes } \\
\text { treadmill training with } 10 \text { minutes } \\
\text { physiotherapy }\end{array}$ & $\begin{array}{l}\text { Primary outcome: } \\
\text { Harris Hip Score: Intervention vs. } \\
\text { control significantly higher ( } p< \\
0.0001) \text { at } 10 \text { days ( } 13.6 \text { points), } 3 \\
\text { months ( } 8.9 \text { points) and } 12 \text { months } \\
\text { (16.5 points). } \\
\text { Secondary outcomes: } \\
\text { No change in walking velocity } \\
\text { between groups } \\
\text { Mean interval to abandon crutches } \\
3.2 \text { wks intervention vs } 7.9 \text { wks } \\
\text { control } \\
\text { At end of } 10 / 7 \text { program, for } \\
\text { intervention group: } \\
\text { Hip extension deficit } 6.8^{\circ} \text { less } \\
\text { Gait symmetry } 10 \% \text { greater } \\
\text { Affected hip abductor stronger } \\
\text { Amplitude of gluteus medius activity } \\
41.5 \% \text { greater (ALL above } p< \\
0.0001 \text { ) } \\
\text { Above differences persisted at } 3 \text { and } \\
12 \text { months }\end{array}$ & None recorded & $37.5 \%$ drop out rate at 1 year \\
\hline
\end{tabular}




\begin{tabular}{|c|c|c|c|c|c|c|c|c|}
\hline $\begin{array}{l}\text { Husby et } \\
\text { al., } 2009 \\
{[24]}\end{array}$ & $\begin{array}{l}\text { Control }(n= \\
12) \\
\text { Intervention } \\
(n=12)\end{array}$ & Centre & $\begin{array}{l}5 \\
\text { weeks }\end{array}$ & $\begin{array}{l}\text { Within 1st } \\
\text { week } \\
\text { postoperatively }\end{array}$ & $\begin{array}{l}\text { Control: Inpatient rehabilitation } \\
\text { treatment with sling exercise therapy } \\
\text { in hip abduction/adduction, flexion/ } \\
\text { extension; low resistance exercises } \\
\text { for } 1 \text { hour, } 5 \text { days a week for } 4 \\
\text { weeks. Patients discharged before } 4 \\
\text { weeks had outpatient treatment } 3 \times / \\
\text { week and were encouraged to do } \\
\text { exercises at home } 2 \times / \text { week. } \\
\text { Intervention: Above regime and } 5 \\
\text { training bouts per week: } \sim 10 \text { minute } \\
\text { warm up then stationary cycling at } \\
\text { Vo } 2 \text { max } 50 \% \text {; strength training with } \\
2 \text { exercises leg press and hip } \\
\text { abduction on operated leg only. } 4 \\
\text { series with rest periods of } 2 \text { minutes }\end{array}$ & $\begin{array}{l}\text { Bilateral leg press: } 40.9 \% \\
\text { improvement in intervention vs. } \\
\text { control group at } 5 \text { weeks }(p<0.002) \text {. } \\
\text { Operated leg strength increased by } \\
65.2 \% \text { vs. control at } 5 \text { weeks ( } p< \\
0.002 \text { ) } \\
\text { Abductor strength in operated leg } \\
87 \% \text { more pronounced in } \\
\text { intervention vs control at } 5 \text { weeks ( } p \\
<0.002 \text { ). } \\
\text { No difference in gait parameters and } \\
\text { health related quality of life } \\
\text { outcomes (SF36) at } 5 \text { weeks } \\
\text { between groups. } \\
\text { For work efficiency, the intervention } \\
\text { lowered the heart rate by } 11.4 \% \\
\text { relative to the control group at } 5 \\
\text { weeks and it also led to a } 32.3 \% \\
\text { trends towards better work efficiency } \\
(p=0.065 \text { ) after } 5 \text { weeks. }\end{array}$ & None recorded & $\begin{array}{l}\text { Lack of adequate sample size to } \\
\text { demonstrate significant differences } \\
\text { in parameters used to assess work } \\
\text { efficiency }\end{array}$ \\
\hline Article & $\begin{array}{l}\text { Number of } \\
\text { participants }\end{array}$ & Site & $\begin{array}{l}\text { Follow } \\
\text { up } \\
\text { period }\end{array}$ & $\begin{array}{l}\text { Interval from } \\
\text { surgery to } \\
\text { intervention }\end{array}$ & Exercise intervention & Outcome measures & $\begin{array}{l}\text { Dislocation } \\
\text { rate }\end{array}$ & Limitations \\
\hline $\begin{array}{l}\text { Jan et al., } \\
2004 \\
{[25]}\end{array}$ & $\begin{array}{l}\text { Control }(n= \\
29), \\
\text { Intervention } \\
(n=29\end{array}$ & Home & $\begin{array}{l}12 \\
\text { weeks }\end{array}$ & $\begin{array}{l}\text { At least } 1.5 \\
\text { years }\end{array}$ & $\begin{array}{l}\text { Control group: no exercises } \\
\text { Intervention group: } 12 \text { week exercise } \\
\text { program inclusive of hip flexion } \\
\text { range of motion, isotonic } \\
\text { strengthening of hip flexors, } \\
\text { extensors and abductors using ankle } \\
\text { weights, walking + weekly telephone } \\
\text { calls }\end{array}$ & $\begin{array}{l}\text { Strength measured with an isokinetic } \\
\text { dynamometer. } \\
\text { High compliance intervention group, } \\
\mathrm{n}=13(>50 \% \text { adherence to } \\
\text { protocol), showed significant } \\
\text { improvement in strength of hip } \\
\text { abductors, flexors and extensors on } \\
\text { both operated and non-operated } \\
\text { legs, as well as greater walking } \\
\text { speed and functional activity } \\
\text { component of Harris hip score } \\
\text { compared to low compliance group, } \\
\mathrm{n}=12 \text { and normal control. }\end{array}$ & None recorded & $\begin{array}{l}\text { Subjects in the intervention group } \\
\text { were not allowed to visit any } \\
\text { physiotherapy department but if } \\
\text { they raised issues with the program, } \\
\text { they were invited to return to the } \\
\text { laboratory for further instructions. No } \\
\text { detail is given as to what proportion } \\
\text { of the cohort required this and how } \\
\text { often. }\end{array}$ \\
\hline $\begin{array}{l}\text { Jesudason } \\
\text { et al., } 2002 \\
{[26]}\end{array}$ & $\begin{array}{l}\text { Intervention } \\
\mathrm{n}=21 \\
\text { Control } \mathrm{n}= \\
21\end{array}$ & Centre & 7 days & $\begin{array}{l}\text { 1st post- } \\
\text { operative day }\end{array}$ & $\begin{array}{l}\text { Control group: Standard protocol for } \\
\text { mobilisation, progression of mobility } \\
\text { as determined by treating } \\
\text { physiotherapist } \\
\text { Exercise group: Bed exercises; hip, } \\
\text { knee, ankle range of movement } \\
\text { exercises. Progressed from } 5 \\
\text { repetitions once a day to } 10 \\
\text { repetitions as tolerated } 2-3 \text { times per } \\
\text { day }\end{array}$ & $\begin{array}{l}\text { Pain severity: } \\
\text { Significant } \downarrow \text { in pain }(p=0.01 \text { ) in } \\
\text { both groups from days 3-7 post-op. } \\
\text { No significant difference in both } \\
\text { groups in terms of hip flexion, } \\
\text { abduction range of movement, } \\
\text { function using the ILOA scale, or } \\
\text { length of stay at } 3 \text { or } 7 \text { days post- } \\
\text { operatively. }\end{array}$ & None recorded & $\begin{array}{l}\text { Short intervention } \\
\text { Short period of follow up } \\
\text { No objective assessment of muscle } \\
\text { strength }\end{array}$ \\
\hline
\end{tabular}


Table 1 Characteristics of randomised controlled trials on hip arthroplasty rehabilitation interventions to improve functional outcome (Continued)

\begin{tabular}{|c|c|c|c|c|c|c|c|c|}
\hline $\begin{array}{l}\text { Liebs et al., } \\
2010 \\
{[27]}\end{array}$ & $\begin{array}{l}\text { Hip } \\
\text { arthroplasty } \\
\text { subgroup. } \\
\text { Control } \mathrm{n}= \\
\text { 104; } \\
\text { Intervention } \\
\mathrm{n}=99\end{array}$ & Centre & $\begin{array}{l}24 \\
\text { months }\end{array}$ & $\begin{array}{l}2 \text { weeks } \\
\text { postoperatively }\end{array}$ & $\begin{array}{l}\text { Control: No ergometer cycling } \\
\text { Intervention: Physiotherapist guided } \\
\text { sessions with ergometer initially. } \\
\text { Sessions } 3 / \text { week for } \geq 3 \text { weeks. } \\
\text { All patients: standard program of } \\
\text { physiotherapy including range of } \\
\text { motion exercises, ADL based } \\
\text { movements and walking on stairs } \\
\text { and uneven surfaces }\end{array}$ & $\begin{array}{l}\text { Primary outcomes: } \\
\text { WOMAC function subscale: } \\
\text { Intervention more improved than } \\
\text { control at } 3 \text { months ( } 16.4 \text { vs. } 21.6 \text { ( } p \\
=0.046) \text { ) and } 24 \text { months ( } 9 \text { vs. } 14.7 \\
(p=0.019) \text { ) } \\
\text { Secondary outcomes } \\
\text { WOMAC stiffness subscale: } \\
\text { Intervention more improved than } \\
\text { control at } 24 \text { months ( } 13.4 \text { vs. } 18.6 \text { ( } p \\
=0.047) \text { ) } \\
\text { WOMAC pain: Intervention more } \\
\text { improved than control at } 3 \text { months } \\
\text { (11.1 vs. } 15.9 \text { ( } p=0.049) \text { ) } \\
\text { Improvements also noted in } \\
\text { intervention vs control in Lequesne } \\
\text { hip and knee score (at } 24 \text { months), } \\
\text { SF36 (6 and } 24 \text { months) and patient } \\
\text { satisfaction ( } 92 \% \text { vs. } 80 \%)\end{array}$ & $\begin{array}{l}1 \text { dislocation in } \\
\text { both groups }\end{array}$ & $\begin{array}{l}\text { Mixed hip and knee arthroplasty } \\
\text { population } \\
77 \% \text { follow up at } 24 \text { months }\end{array}$ \\
\hline Article & $\begin{array}{l}\text { Number of } \\
\text { participants }\end{array}$ & Site & $\begin{array}{l}\text { Follow } \\
\text { up } \\
\text { period }\end{array}$ & $\begin{array}{l}\text { Interval from } \\
\text { surgery to } \\
\text { intervention }\end{array}$ & Exercise intervention & Outcome measures & $\begin{array}{l}\text { Dislocation } \\
\text { rate }\end{array}$ & Limitations \\
\hline $\begin{array}{l}\text { Mahomed } \\
\text { et al., } 2008 \\
\text { [28] }\end{array}$ & $\begin{array}{l}\text { Home based } \\
\mathrm{n}=115 \\
\text { Centre based } \\
\mathrm{n}=119\end{array}$ & $\begin{array}{l}\text { Home/ } \\
\text { Centre }\end{array}$ & $\begin{array}{l}12 \\
\text { months }\end{array}$ & $\begin{array}{l}\text { On discharge } \\
\text { from hospital }\end{array}$ & $\begin{array}{l}\text { All patients: standard physiotherapy } \\
\text { regimen: deep breathing, coughing, } \\
\text { active and assisted bed/chair gait } \\
\text { training } \\
\text { Home regime: Referral to } \\
\text { community team: nursing, home } \\
\text { support etc. Patients discharged } \\
\text { when functionally improved as } \\
\text { determined by attending therapist } \\
\text { Centre-regime: } 14 \text { day stay in rehab } \\
\text { centre with established pathway } \\
\text { (regime not specified) }\end{array}$ & $\begin{array}{l}\text { Primary outcomes: } \\
\text { WOMAC function subscale: no } \\
\text { difference between groups at } 3 \text { and } \\
12 \text { months } \\
\text { Hip and Knee satisfaction scale: no } \\
\text { difference between groups at } 3 \text { and } \\
12 \text { months } \\
\text { SF36 short form: no difference at } 3 \\
\text { and } 12 \text { months } \\
\text { Impatient rehabilitation more } \\
\text { expensive than home based ( } \$ 14531 \\
\text { vs. } \$ 11082 \text { ) }\end{array}$ & $\begin{array}{l}2 \% \text { dislocation } \\
\text { rate in both } \\
\text { intervention and } \\
\text { control groups }\end{array}$ & $\begin{array}{l}\text { Hip and knee arthroplasty patients } \\
\text { included. No specific detail given for } \\
\text { hip population }\end{array}$ \\
\hline $\begin{array}{l}\text { Munin et } \\
\text { al., } 1998 \\
{[29]}\end{array}$ & $\begin{array}{l}\text { Mixed hip } \\
\text { and knee } \\
\text { arthroplasty. } \\
\text { Total } \mathrm{n}=70 \\
\text { Hip cohort: } \\
\text { Control } \mathrm{n}= \\
12 \text {; } \\
\text { Intervention } \\
\mathrm{n}=14\end{array}$ & Centre & $\begin{array}{l}16 \\
\text { weeks }\end{array}$ & $\begin{array}{l}\text { Immediate } \\
\text { post-operative } \\
\text { period }\end{array}$ & $\begin{array}{l}\text { Intervention group: Commenced } \\
\text { rehabilitation protocol at } 3 \text { days } \\
\text { post-op } \\
\text { Control group: Commenced } \\
\text { rehabilitation protocol at } 7 \text { days } \\
\text { post-op. }\end{array}$ & $\begin{array}{l}\text { Median length of stay: intervention } \\
12.2 \text { days vs. control } 14.8 \text { days } \\
\text { Cost of surgery and rehabilitation } \\
\text { lower for intervention ( } \$ 28256) \text { than } \\
\text { control ( } \$ 29437) \text {. } \\
\text { RAND } 36 \text { functional self assessment: } \\
\text { No difference between both groups } \\
\text { through the follow-up period }\end{array}$ & $\begin{array}{l}1 \text { dislocation } \\
\text { each in control } \\
\text { and intervention } \\
\text { groups }\end{array}$ & $\begin{array}{l}\text { Mixed hip and knee arthroplasty } \\
\text { population } \\
\text { Analysing both hips and knees } \\
\text { together, the intervention group } \\
\text { shows more rapid attainment of } \\
\text { short term functional milestones } \\
\text { such as ambulation, walking distance } \\
\text { and stair climbing ability at 6-10 } \\
\text { days post-op. No difference existed } \\
\text { for stratifying patients to type of } \\
\text { surgery. }\end{array}$ \\
\hline Article & $\begin{array}{l}\text { Number of } \\
\text { participants }\end{array}$ & Site & $\begin{array}{l}\text { Follow } \\
\text { up } \\
\text { period }\end{array}$ & $\begin{array}{l}\text { Interval from } \\
\text { surgery to } \\
\text { intervention }\end{array}$ & Exercise intervention & Outcome measures & $\begin{array}{l}\text { Dislocation } \\
\text { rate }\end{array}$ & Limitations \\
\hline $\begin{array}{l}\text { Rahmann } \\
\text { et al., } 2009 \\
\text { [30] }\end{array}$ & $\begin{array}{l}\text { Control } n= \\
17, \text { Aquatic } \\
\text { group } n= \\
18, \text { Water } \\
\text { exercises } n= \\
19\end{array}$ & Centre & $\begin{array}{l}180 \\
\text { days }\end{array}$ & $\begin{array}{l}\text { From post-op } \\
\text { days } 4-10\end{array}$ & $\begin{array}{l}\text { All patients: Standard physiotherapy } \\
\text { x1/day } \\
\text { Ward control: as above } \\
\text { Water exercise group: General } \\
\text { exercises in water but not targeted } \\
\text { at specific functional retraining in } \\
\text { the aquatic environment ( } 40 \text { minutes } \\
\text { once daily till discharge) } \\
\text { Aquatic group: Hip abductor/ } \\
\text { adductor exercises with increasing } \\
\text { progression- squat, heel raises in } \\
\text { various positions in pool (40 minutes } \\
\text { once daily till discharge) }\end{array}$ & $\begin{array}{l}\text { Hip subgroup: } \\
\text { No significant difference across the } 3 \\
\text { groups for primary outcomes such } \\
\text { as hip abductor strength, } 10 \mathrm{~m} \text { walk, } \\
\text { WOMAC score and secondary } \\
\text { outcomes such as timed up and go, } \\
\text { quadriceps strength. }\end{array}$ & None recorded & $\begin{array}{l}\text { Mixed group of hip and knee } \\
\text { arthroplasty patients } \\
\text { Small number of participants }\end{array}$ \\
\hline
\end{tabular}


Table 1 Characteristics of randomised controlled trials on hip arthroplasty rehabilitation interventions to improve functional outcome (Continued)

\begin{tabular}{|c|c|c|c|c|c|c|c|c|}
\hline $\begin{array}{l}\text { Smith et al., } \\
2008 \\
{[31]}\end{array}$ & $\begin{array}{l}\text { Control } \mathrm{n}= \\
30 \\
\text { Intervention } \\
\mathrm{n}=30\end{array}$ & Centre & $\begin{array}{l}6 \\
\text { weeks }\end{array}$ & $\begin{array}{l}\text { Immediate } \\
\text { post-operative } \\
\text { period }\end{array}$ & $\begin{array}{l}\text { Control group: Standard gait re- } \\
\text { education protocol from post- } \\
\text { operative day } 1 \\
\text { Intervention group: Gait re-education } \\
\text { with programme of bed exercises } \\
\text { from day } 1 \text { including; active hip } \\
\text { flexion, ankle dorsi/plantarflexion, } \\
\text { static quads and gluteal exercises. } 10 \\
\text { repetitions each, } 5 \text { times a day } \\
\text { during hospital stay. Patients } \\
\text { encouraged to continue same } \\
\text { regime on discharge }\end{array}$ & $\begin{array}{l}\text { lowa level of assistance (ILOA): } \\
\text { Significant improvement from } \\
\text { baseline in both groups but no } \\
\text { difference in both groups at } 3 \text { days } \\
\text { and } 6 \text { weeks } \\
\text { SF12: No difference in both groups }\end{array}$ & $\begin{array}{l}\text { At week 6, } 1 \\
\text { dislocation in } \\
\text { control group; } \\
\text { no dislocations } \\
\text { recorded in } \\
\text { intervention } \\
\text { group }\end{array}$ & $\begin{array}{l}\text { No concealed allocation of } \\
\text { randomisation so possible selection } \\
\text { bias } \\
\text { No objective assessment of hip } \\
\text { strength performed }\end{array}$ \\
\hline $\begin{array}{l}\text { Stockton et } \\
\text { al., } 2009 \\
{[32]}\end{array}$ & $\begin{array}{l}\text { Control } \mathrm{n}= \\
27 \\
\text { Intervention } \\
\mathrm{n}=30\end{array}$ & Centre & 6 days & $\begin{array}{l}\text { Immediate } \\
\text { post-operative } \\
\text { period }\end{array}$ & $\begin{array}{l}\text { Control group: Once daily } \\
\text { physiotherapy including mobilisation } \\
\text { exercises and transfer practice. } \\
\text { Encouragement to perform } 4 \times \text { daily } \\
\text { till independently mobile } \\
\text { Intervention group: } 2 \text { physiotherapy } \\
\text { sessions per day. Similar protocol to } \\
\text { above }\end{array}$ & $\begin{array}{l}\text { Length of stay: } \\
\text { No significant difference } \\
\text {-Intervention ( } 8.2 \text { days) vs control ( } 8 \\
\text { days) } \\
\text { ILOA: Significant difference at } 3 \text { days } \\
\text { (intervention } 28.5 \text { vs control } 32.2 \text { ( } \\
=0.041 \text { ) but not at } 7 \text { days } \\
\text { (intervention } 18.2 \text { vs control 20.6) }\end{array}$ & None recorded & $\begin{array}{l}\text { Length of follow up } \\
\text { No objective measurement of } \\
\text { muscle strength }\end{array}$ \\
\hline Article & $\begin{array}{l}\text { Number of } \\
\text { participants }\end{array}$ & Site & $\begin{array}{l}\text { Follow } \\
\text { up } \\
\text { period }\end{array}$ & $\begin{array}{l}\text { Interval from } \\
\text { surgery to } \\
\text { intervention }\end{array}$ & Exercise intervention & Outcome measures & $\begin{array}{l}\text { Dislocation } \\
\text { rate }\end{array}$ & Limitations \\
\hline $\begin{array}{l}\text { Suetta et } \\
\text { al., } 2004 \\
\text { [14] }\end{array}$ & $\begin{array}{l}\text { Total } n=36 \text {; } \\
\text { Standard } \\
\text { rehabilitation } \\
(\mathrm{SR}) \mathrm{n}=12 \text {, } \\
\text { Electrical } \\
\text { stimulation } \\
\text { (ES) } n=11 \text {, } \\
\text { Resistance } \\
\text { training } \\
(\mathrm{RT}) \mathrm{n}=13\end{array}$ & $\begin{array}{l}\text { Centre/ } \\
\text { Home }\end{array}$ & $\begin{array}{l}12 \\
\text { weeks }\end{array}$ & $\begin{array}{l}\text { Immediate } \\
\text { post-operative } \\
\text { period }\end{array}$ & $\begin{array}{l}\text { SR: } 15 \text { exercises in } 2 \text { parts. } 1^{\text {st }} \text { part } 6 \\
\text { bed exercises; } 2^{\text {nd }} \text { part knee } \\
\text { extensions in seated position and } \\
\text { hip abduction, knee flexion, step } \\
\text { training and calf stretching while } \\
\text { standing. The attending } \\
\text { physiotherapist added ambulation } \\
\text { and transfer during the inpatient } \\
\text { stay. Exercise was encouraged in the } \\
\text { home setting } 2 \times / \text { day and } \\
\text { attendance was arranged at a } \\
\text { physiotherapy department once a } \\
\text { week. } \\
\text { NM: Electrodes placed over } \\
\text { quadriceps of operated leg } 5 \mathrm{~cm} \\
\text { below inguinal ligament and } 5 \mathrm{~cm} \\
\text { above patella. Pulse rate } 40 \mathrm{~Hz} \text {, pulse } \\
\text { width } 250 \mu \text { s, stimulation } ~ 10 \mathrm{~s} \text { with } \\
20 \mathrm{~s} \text { of rest. Total stimulation } 1 \text { hour } \\
\text { per day for } 12 \text { weeks. } \\
\text { RT: Unilateral progressive resistance } \\
\text { training for quadriceps of operated } \\
\text { leg. Exercises included knee } \\
\text { extension in seated position with } \\
\text { sandbags on ankles, leg presses in } \\
\text { supine position, supervised by } \\
\text { trained physiotherapist. Intensity } \\
\text { increased from } 50 \% \text { of } 1 \text { RM in week } \\
1 \text { to } 65 \% 1 \text { RM weeks } 2-4,70 \% 1 \text { RM } \\
\text { weeks } 5,6 \text { and } 80 \% 1 \text { RM last } 6 \\
\text { weeks. For each session patients } \\
\text { performed } 3-5 \text { sets of } 10 \text { repetitions } \\
\text { during weeks } 1-5 \text { and } 2-5 \text { sets of } 8 \\
\text { repetitions weeks } 6-12 \text {. }\end{array}$ & $\begin{array}{l}\text { Length of Stay: } \\
\text { RT led to the shortest length of stay } \\
\text { compared to ES and SR ( } 10 \pm 2.4 \\
\text { days vs. } 12 \pm 2.8 \text { and } 16 \pm 7.2 \\
\text { respectively). The difference ( } 37 \% \text { ) } \\
\text { between RT and SR was statistically } \\
\text { significant ( } p<0.05 \text { ). } \\
\text { Functional performance: } \\
\text { Gait speed: RT } \uparrow \text { maximal gait speed } \\
\text { by } 30 \% \text { at } 12 \text { weeks ( } p<0.001 \text { ) } \\
\text { whilst ES increases it by } 19 \% \text { ( } p< \\
\text { 0.05). No increase was seen in the SR } \\
\text { group. } \\
\text { Sit to stand: RT } \uparrow 30 \% \text {, ES } \uparrow 21 \% \\
\text { (both } p<0.001 \text { ) at } 12 \text { weeks. SR no } \\
\text { improvement. } \\
\text { Stair Climb: RT } \uparrow 28 \% \text { ( } p<0.005 \text { ), ES } \\
21 \% \text { ( } p<0.001) \text {. SR no } \\
\text { improvement. } \\
\text { Quadriceps cross sectional area } \\
\text { (CSA): } \\
\text { At } 12 \text { weeks, CSA of operated leg } \\
\text { was } \uparrow 12 \% \text { in RT group, } \uparrow 7 \% \text { in ES } \\
\text { group and } \downarrow 9 \% \text { in SR group (all } p< \\
0.05 \text { ). The non-operated leg was } \\
\text { unaffected in all the groups. } \\
\text { Peak torque on operated leg at } 12 \\
\text { weeks was } \uparrow 22 \% \text { in RT group ( } p< \\
0.05 \text { ) and unchanged in ES and SR } \\
\text { groups. No change was noted in } \\
\text { any of the groups for the non- } \\
\text { operated leg. }\end{array}$ & None recorded & $\begin{array}{l}\text { No assessment of compliance in the } \\
\text { SR group } \\
\text { No documentation as to whether ES } \\
\text { group received additional support } \\
\text { for ambulation and transfer } \\
\text { No use of subjective outcome } \\
\text { measures } \\
\text { Length of stay assessed was } \\
\text { cumulative: did not discriminate } \\
\text { between acute surgical inpatient } \\
\text { stay and rehabilitation centre length } \\
\text { of stay }\end{array}$ \\
\hline
\end{tabular}


Table 1 Characteristics of randomised controlled trials on hip arthroplasty rehabilitation interventions to improve functional outcome (Continued)

\begin{tabular}{|c|c|c|c|c|c|c|c|c|}
\hline Article & $\begin{array}{l}\text { Number of } \\
\text { participants }\end{array}$ & Site & $\begin{array}{l}\text { Follow } \\
\text { up } \\
\text { period }\end{array}$ & $\begin{array}{l}\text { Interval from } \\
\text { surgery to } \\
\text { intervention }\end{array}$ & Exercise intervention & Outcome measures & $\begin{array}{l}\text { Dislocation } \\
\text { rate }\end{array}$ & Limitations \\
\hline $\begin{array}{l}\text { Trudelle- } \\
\text { Jackson et } \\
\text { al. } 2004 \\
{[5]}\end{array}$ & $\begin{array}{l}\text { Control } \mathrm{n}= \\
14 \\
\text { Intervention } \\
\mathrm{n}=14\end{array}$ & Home & $\begin{array}{l}8 \\
\text { weeks }\end{array}$ & $\begin{array}{l}\text { 4-12 months } \\
\text { post- } \\
\text { operatively }\end{array}$ & $\begin{array}{l}\text { Control: } 7 \text { basic isometric and active } \\
\text { range of movement exercises } \\
\text { including the glutei, quads, } \\
\text { hamstring sets, ankle pumps, heel } \\
\text { slides, hip abduction in supine } \\
\text { position and hip internal and } \\
\text { external rotation in supine position. } \\
\text { Intervention: Sit to stand, unilateral } \\
\text { heel raises, partial knee bends, 1- } \\
\text { legged standing stance, knee raises } \\
\text { with alternate arm raise, side and } \\
\text { back leg raises in standing, unilateral } \\
\text { pelvic lowering and raising in } \\
\text { standing } \\
\text { Both groups: Progressively increasing } \\
\text { repetitions of exercises encouraged } \\
\text { 3-4/week for } 8 \text { weeks }\end{array}$ & $\begin{array}{l}\text { No difference in fear of falling } \\
\text { between both groups. } \\
\text { Significant increase in following in } \\
\text { the intervention group compared to } \\
\text { control at } 8 \text { weeks: } \\
\text { Hip flexor strength }(\uparrow 47.8 \%) \\
\text { Hip extensor strength }(\uparrow 41.2 \%) \\
\text { Hip abductor strength }(\uparrow 23.4 \%) \\
\text { Postural stability }(\uparrow 36.8 \%)\end{array}$ & None recorded & $\begin{array}{l}\text { Not clear whether the intervention } \\
\text { and control groups both received } \\
\text { the same amount of encouragement } \\
\text { with the visits to increase repetitions } \\
\text { Short follow up period }\end{array}$ \\
\hline
\end{tabular}


to ensure maintenance of a constant relative intensity) (Table 2; 6 out of 6 centre based studies $[14,21,23,24,26,27]$ involving resistance training proved beneficial). The only centre based intervention, by Gremeaux et al., [22], that led to significant improvements in muscle strength without progressive resistance training utilised electrical stimulation. This has been shown by Suetta et al. [14] to not be as efficacious when directly compared to PRT (Table 1). Both home based intervention studies identified in this review $[5,25]$ were carried out in the late phase (> 1 month post-operatively) and led to significant improvements in functional outcome parameters after short periods of follow up (8 and 12 weeks respectively). The other 6 studies reviewed include 2 comparing home and centre based interventions $[20,28]$ and 4 others performed in the early phase in a centre setting [29-32] but without the use of progressive training. Their limitations and findings are as detailed in Table 1.

The follow up periods for the centre based studies varied from 7 days to 24 months (Table 1). In terms of the follow up periods that were longer than the interventions used, Liebs et al. [27] show with their ergometer study that the benefits of a resistance program are sustained for 24 months from surgery which helps to address the functional deficits identified after THR. Mahomed et al. [28] demonstrated that there is not much difference at 1 year between home and centre based post-THR standard physiotherapy interventions in terms of subjective functional outcome (measured with the WOMAC), but there was no progressive training included in the prescribed programs used and this may explain the lack of a significant difference between the groups.

\section{Discussion}

'Standard physiotherapy', (i.e. not involving resistance training) following major surgery enables most patients to regain basal levels of function but leaves them with significant muscle wasting as it lacks the intensity of exercise required to elicit muscle hypertrophy $[14,33]$. The most commonly used rehabilitation regimes for

Table 2 Timing and effects of rehabilitation interventions following hip arthroplasty

\begin{tabular}{|c|c|c|c|c|}
\hline Article & $\begin{array}{l}\text { Intervention } \\
\text { site }\end{array}$ & $\begin{array}{l}\text { Timing of intervention: } \\
\text { Early (>1 month) or late } \\
\text { (>1 month) }\end{array}$ & $\begin{array}{l}\text { Use of progressive } \\
\text { resistance training? } \\
\text { Yes/No }\end{array}$ & $\begin{array}{l}\text { Significant effect of intervention on functional } \\
\text { outcomes measured? } \\
\text { Yes/No }\end{array}$ \\
\hline $\begin{array}{l}\text { Giaquinto et al., } 2008 \\
\text { [21] }\end{array}$ & Centre & Early & Yes & Yes \\
\hline $\begin{array}{l}\text { Husby et al., } 2009 \\
{[24]}\end{array}$ & Centre & Early & Yes & Yes \\
\hline $\begin{array}{l}\text { Galea et al., } 2008 \\
{[20]}\end{array}$ & Home/Centre & Early & No & No \\
\hline $\begin{array}{l}\text { Smith et al., } 2008 \\
\text { [31] }\end{array}$ & Centre & Early & No & No \\
\hline $\begin{array}{l}\text { Rahmann et al., } 2009 \\
\text { [30] }\end{array}$ & Centre & Early & No & No \\
\hline $\begin{array}{l}\text { Liebs et al., } 2010 \\
{[27]}\end{array}$ & Centre & Early & Yes & Yes \\
\hline $\begin{array}{l}\text { Mahomed et al., } \\
2008 \text { [28] }\end{array}$ & Home/Centre & Early & No & No \\
\hline $\begin{array}{l}\text { Hesse et al., } 2003 \\
{[23]}\end{array}$ & Centre & Early & Yes & Yes \\
\hline $\begin{array}{l}\text { Munin et al., } 1998 \\
\text { [29] }\end{array}$ & Centre & Early & No & No \\
\hline $\begin{array}{l}\text { Gremeaux et al., } \\
2008 \text { [22] }\end{array}$ & Centre & Early & No & Yes \\
\hline $\begin{array}{l}\text { Jesudason et al., } \\
2002 \text { [26] }\end{array}$ & Centre & Early & Yes & Yes \\
\hline $\begin{array}{l}\text { Suetta et al., } 2004 \\
\text { [14] }\end{array}$ & Centre & Early & Yes & Yes \\
\hline $\begin{array}{l}\text { Trudelle-Jackson et } \\
\text { al., } 2004 \text { [5] }\end{array}$ & Home & Late & Yes & Yes \\
\hline Jan et al., 2004 [25] & Home & Late & Yes & Yes \\
\hline $\begin{array}{l}\text { Stockton et al., } 2009 \\
\text { [32] }\end{array}$ & Centre & Early & No & No \\
\hline
\end{tabular}


elderly individuals are based on functional types of exercises without external loading. However, this type of intervention not only fails to elicit increases in muscle mass but does not prevent further muscle atrophy [34]. In contrast, high-intensity PRT is an extremely effective and safe method for inducing muscle hypertrophy and increasing muscle strength and subsequently improving functional performance in healthy individuals, those with chronic disease e.g. rheumatoid arthritis [33], and the elderly [35-37]. An unpublished survey from our institution of physiotherapy practice around the UK after THR revealed that $73 \%$ of qualified physiotherapists knew what progressive resistance training entailed but only $32 \%$ used it in their prescribed programmes after THR.

PRT typically elicits positive health and performance adaptations by challenging the skeletal muscles with loads that can be lifted repetitively for 8-15 repetitions maximum (RM) per set before the onset of neuromuscular fatigue i.e. the point at which appropriate technique can no longer be maintained $[3,38]$. PRT sessions are optimal when followed by periods of recovery ranging from 48 to $72 \mathrm{~h}$ to allow for physiological super compensation (i.e. positive adaptation)[39]. To facilitate continued adaptation and avoid the onset of a plateau in physiological adaptation, training intensity (i.e. load) and/or training volume (i.e. the total number of lifts) are progressively increased in line with training response [39]. Health and performance are maintained with continued training [40] and PRT is well established as a safe and beneficial exercise modality for individuals of all ages and fitness levels, including those afflicted with severe chronic illnesses [33,39,41]. Additionally, PRT is particularly beneficial for elderly individuals given its efficacy in counteracting sarcopenia, reducing fat mass, abating osteoporosis, and reversing the many physiological and functional impairments that accrue with age [4-6]. The positive benefits of this method of rehabilitation are evident with the 5 randomised controlled trials identified in this review (Table 2).

A major disadvantage of programs used in the postoperative period following THR is the need for patients to exercise under the supervision of professional staff at a hospital or rehabilitation centre [20]. This makes program delivery expensive due to the high costs associated with supervision and transport. In addition, some THR patients are excluded because of difficulties with mobility [7]. A similar exercise regime that could be performed at home would overcome these cost and logistic implications.

A limitation of the home-based interventions is that follow-up did not extend beyond the end of the exercise interventions periods. Thus, it is not clear whether the benefits evident at the end of the exercise intervention are maintained in the longer term. The other obvious shortcoming is the lateness of the intervention in the home setting and consequently the failure to ameliorate or prevent the exacerbated loss of muscle and function after surgery. A recent systematic review by Di Monaco et al. [42] suggests that the difficulties in THR rehabilitation research are that there is a lack of multicentre clinical trials with large sample sizes to inform the design of optimal physical exercise programs.

It follows that with the adverse impact of major surgery on muscle mass and therefore strength and function, being able to provide an intervention in the early post-operative period is essential. The intervention should also obviate the problems of cost and transport that supervised, centre-based rehabilitation programs necessarily involve. Providing patients with the impetus to rehabilitate themselves with minimal supervision in their home environments may be the answer.

A major concern with orthopaedic surgeons is dislocation of the hip arthroplasty (incidence after primary THR of $1-9.5 \%$ [43] on patient mobilisation and the instructions patients have to adhere to afterwards take this into account. These include a restriction of hip flexion to less than $90^{\circ}$, no crossing of the legs, and elevation of toilet seats and chairs in the house etc. From this systematic review of 15 randomised controlled trials, the dislocation rate in the pooled sample of patients who underwent the interventions described was $0.77 \%$ (4 recorded dislocations in a pooled sample of $n$ $=516$ ) whilst the rate in patients who were in the normal control groups was 1\% (5 recorded dislocations in a pooled sample of $n=505$ ). Thus, it is safe to conclude that these exercise programmes are not associated with an increased risk of dislocation.

\section{Conclusions}

Total hip replacement surgery provides good relief for patients' pain but fails to fully restore physical function. This systematic review demonstrates that significant improvements in muscle strength and function are achievable with PRT. Regardless of the timing of the intervention, PRT appears to have a significant benefit on patient function following THR. Late PRT interventions do work and are safe, and they have been performed mainly in the home setting but the studies done have short periods of follow up and have a further limitation of the pre-existing functional deficit due to the timing post-operatively. Early PRT regimes identified in the studies reviewed in this article have shown the need for a centre-based approach and this has demonstrable benefit but there are issues of high costs of transport and supervision. Early home based PRT studies that are effective and safe; with adequate follow-up after THR surgery would potentially address these issues. 


\section{Funding}

This work was supported by a grant from the Betsi Cadwaladr University Health Board Small Grants Committee.

\section{Ethical standards}

There were no ethical considerations in the preparation of this review

\section{Author details}

${ }^{1}$ School of Medical Sciences (SMS)/School of Sport Health and Exercise Science (SSHES), Bangor University, Penrallt Road, Bangor LL57 2AS, UK. ${ }^{2} S c h o o l$ of Sport, Health and Exercise Science (SSHES), Bangor University, Holyhead Road, Bangor LL57 2PZ, UK. ${ }^{3}$ Department of Orthopaedics, Ysbyty Gwynedd, Betsi Cadwaladr University Health Board, Penrhosgarnedd, Bangor LL57 2PW, UK

\section{Authors' contributions}

TO carried out the review of the literature, wrote and made changes to the final version of the manuscript as advised by AL, PM and JA (Academic supervisors). All authors read and approved the final manuscript

\section{Competing interests}

There are no competing interests to declare in the preparation of this manuscript.

Received: 17 March 2011 Accepted: 7 February 2012

Published: 7 February 2012

\section{References}

1. Felson DT, Lawrence RC, Dieppe PA, Hirsch R, Helmick CG, Jordan JM, et al: Osteoarthritis: new insights. Part 1: the disease and its risk factors. Ann Intern Med 2000, 133(8):635-646.

2. Dawson J, Linsell L, Zondervan K, Rose P, Randall T, Carr A, et al: Epidemiology of hip and knee pain and its impact on overall health status in older adults. Rheumatology (Oxford) 2004, 43(4):497-504.

3. Di Domenica F, Sarzi-Puttini P, Cazzola M, Atzeni F, Cappadonia C, Caserta A, et al: Physical and rehabilitative approaches in osteoarthritis. Semin Arthritis Rheum 2005, 34(6 Suppl 2):62-69.

4. National Joint Registry for England and Wales. 7th Annual Clinical Report 2010, Accessed 20/09/10.

5. Trudelle-Jackson E, Smith SS: Effects of a late-phase exercise program after total hip arthroplasty: a randomized controlled trial. Arch Phys Med Rehabil 2004, 85(7):1056-1062

6. Kennedy DM, Hanna SE, Stratford PW, Wessel J, Gollish JD: Preoperative function and gender predict pattern of functional recovery after hip and knee arthroplasty. J Arthroplasty 2006, 21(4):559-566

7. Barber TC, Roger DJ, Goodman SB, Schurman DJ: Early outcome of total hip arthroplasty using the direct lateral vs the posterior surgical approach. Orthopedics 1996, 19(10):873-875.

8. Trudelle-Jackson E, Emerson R, Smith S: Outcomes of total hip arthroplasty: a study of patients one year postsurgery. $J$ Orthop Sports Phys Ther 2002, 32(6):260-267.

9. Shih CH, Du YK, Lin YH, Wu CC: Muscular recovery around the hip joint after total hip arthroplasty. Clin Orthop Relat Res 1994, 302:115-120.

10. Long WT, Dorr LD, Healy B, Perry J: Functional recovery of noncemented total hip arthroplasty. Clin Orthop Relat Res 1993, 288:73-77.

11. Rasch A, Bystrom AH, Dalen N, Martinez-Carranza N, Berg HE: Persisting muscle atrophy two years after replacement of the hip. J Bone Joint Surg Br 2009, 91(5):583-588.

12. Lang TF, Cauley J, Tylavsky F, Bauer D, Cummings S, Harris T, et al: Computed Tomography Measurements of Thigh Muscle Cross-Sectional Area and Attenuation Coefficient Predict Hip Fracture: The Health. $J$ Bone Miner Res: Aging and Body Composition Study 2009.

13. Rantanen T, Avlund K, Suominen H, Schroll M, Frandin K, Pertti E: Muscle strength as a predictor of onset of ADL dependence in people aged 75 years. Aging Clin Exp Res 2002, 14(3 Suppl):10-15.
14. Suetta C, Magnusson SP, Rosted A, Aagaard P, Jakobsen AK, Larsen LH, et al: Resistance training in the early postoperative phase reduces hospitalization and leads to muscle hypertrophy in elderly hip surgery patients-a controlled, randomized study. J Am Geriatr Soc 2004, 52(12):2016-2022.

15. Wang AW, Gilbey HJ, Ackland TR: Perioperative exercise programs improve early return of ambulatory function after total hip arthroplasty: a randomized, controlled trial. Am J Phys Med Rehabil 2002, 81(11):801-806.

16. Herndon JH, Hwang R, Bozic KJ: Healthcare technology and technology assessment. Eur Spine J 2007, 16(8):1293-1302.

17. Husted H, Holm G, Jacobsen S: Predictors of length of stay and patient satisfaction after hip and knee replacement surgery: fast-track experience in 712 patients. Acta Orthop 2008, 79(2):168-173.

18. Epstein $A M$, Read $J L$, Hoefer M: The relation of body weight to length of stay and charges for hospital services for patients undergoing elective surgery: a study of two procedures. Am J Public Health 1987, 77(8):993-997.

19. Wigerstad-Lossing I, Grimby G, Jonsson T, Morelli B, Peterson L, Renstrom P: Effects of electrical muscle stimulation combined with voluntary contractions after knee ligament surgery. Med Sci Sports Exerc 1988, 20(1):93-98.

20. Galea MP, Levinger P, Lythgo N, Cimoli C, Weller R, Tully E, et al: A targeted home- and center-based exercise program for people after total hip replacement: a randomized clinical trial. Arch Phys Med Rehabil 2008, 89(8):1442-1447

21. Giaquinto S, Ciotola E, Dall'armi V, Margutti F: Hydrotherapy after total hip arthroplasty: a follow-up study. Arch Gerontol Geriatr 2010, 50(1):92-95.

22. Gremeaux V, Renault J, Pardon L, Deley G, Lepers R, Casillas JM: Lowfrequency electric muscle stimulation combined with physical therapy after total hip arthroplasty for hip osteoarthritis in elderly patients: a randomized controlled trial. Arch Phys Med Rehabil 2008, 89(12):2265-2273.

23. Hesse S, Werner C, Seibel H, von Frankenberg S, Kappel EM, Kirker S, et al: Treadmill training with partial body-weight support after total hip arthroplasty: a randomized controlled trial. Arch Phys Med Rehabil 2003, 84(12):1767-1773.

24. Husby VS, Helgerud J, Bjorgen S, Husby OS, Benum P, Hoff J: Early maximal strength training is an efficient treatment for patients operated with total hip arthroplasty. Arch Phys Med Rehabil 2009, 90(10):1658-1667.

25. Jan MH, Hung JY, Lin JC, Wang SF, Liu TK, Tang PF: Effects of a home program on strength, walking speed, and function after total hip replacement. Arch Phys Med Rehabil 2004, 85(12):1943-1951.

26. Jesudason C, Stiller K: Are bed exercises necessary following hip arthroplasty? Aust J Physiother 2002, 48(2):73-81.

27. Liebs TR, Herzberg W, Ruther W, Haasters J, Russlies M, Hassenpflug J: Ergometer cycling after hip or knee replacement surgery: a randomized controlled trial. J Bone Joint Surg Am 2010, 92(4):814-822.

28. Mahomed NN, Davis AM, Hawker G, Badley E, Davey JR, Syed KA, et al: Inpatient compared with home-based rehabilitation following primary unilateral total hip or knee replacement: a randomized controlled trial. J Bone Joint Surg Am 2008, 90(8):1673-1680.

29. Munin MC, Rudy TE, Glynn NW, Crossett LS, Rubash HE: Early inpatient rehabilitation after elective hip and knee arthroplasty. JAMA 1998, 279(11):847-852.

30. Rahmann AE, Brauer SG, Nitz JC: A specific inpatient aquatic physiotherapy program improves strength after total hip or knee replacement surgery: a randomized controlled trial. Arch Phys Med Rehabil 2009, 90(5):745-755.

31. Smith TO, Mann CJV, Clark A, Donell ST: Bed exercises following total hip replacement: a randomised controlled trial. Physiotherapy 2008, 94(4):286-291.

32. Stockton KA, Mengersen KA: Effect of multiple physiotherapy sessions on functional outcomes in the initial postoperative period after primary total hip replacement: a randomized controlled trial. Arch Phys Med Rehabil 2009, 90(10):1652-1657.

33. Lemmey AB, Marcora SM, Chester K, Wilson S, Casanova F, Maddison PJ: Effects of high-intensity resistance training in patients with rheumatoid arthritis: a randomized controlled trial. Arthritis Rheum 2009, 61(12):1726-1734. 
34. Reardon K, Galea M, Dennett X, Choong P, Byrne E: Quadriceps muscle wasting persists 5 months after total hip arthroplasty for osteoarthritis of the hip: a pilot study. Intern Med J 2001, 31(1):7-14.

35. Harridge SD, Kryger A, Stensgaard A: Knee extensor strength, activation, and size in very elderly people following strength training. Muscle Nerve 1999, 22(7):831-839.

36. Hauer K, Rost B, Rutschle K, Opitz H, Specht N, Bartsch P, et al: Exercise training for rehabilitation and secondary prevention of falls in geriatric patients with a history of injurious falls. J Am Geriatr Soc 2001, 49(1):10-20.

37. Hauer K, Specht N, Schuler M, Bartsch P, Oster P: Intensive physical training in geriatric patients after severe falls and hip surgery. Age Ageing 2002, 31(1):49-57.

38. American College of Sports Medicine Position Stand: The recommended quantity and quality of exercise for developing and maintaining cardiorespiratory and muscular fitness, and flexibility in healthy adults. Med Sci Sports Exerc 1998, 30(6):975-991.

39. Cheema B, Abas H, Smith B, O'Sullivan A, Chan M, Patwardhan A, et al: Progressive exercise for anabolism in kidney disease (PEAK): a randomized, controlled trial of resistance training during hemodialysis. J Am Soc Nephrol 2007, 18(5):1594-1601.

40. Evans WJ: Exercise training guidelines for the elderly. Med Sci Sports Exerc 1999, 31(1):12-17.

41. Singh MA: Exercise comes of age: rationale and recommendations for a geriatric exercise prescription. J Gerontol A Biol Sci Med Sci 2002, 57(5): M262-82.

42. Di Monaco M, Vallero F, Tappero R, Cavanna A: Rehabilitation after total hip arthroplasty: a systematic review of controlled trials on physical exercise programs. Eur J Phys Rehabil Med 2009, 45(3):303-317.

43. Hedlundh $\mathrm{U}$, Hybbinette $\mathrm{CH}$, Fredin $\mathrm{H}$ : Influence of surgical approach on dislocations after Charnley hip arthroplasty. J Arthroplasty 1995, 10(5):609-614

doi:10.1186/1758-2555-4-5

Cite this article as: Okoro et al:: An appraisal of rehabilitation regimes used for improving functional outcome after total hip replacement surgery. Sports Medicine, Arthroscopy, Rehabilitation, Therapy \& Technology 2012 4:5.

\section{Submit your next manuscript to BioMed Central and take full advantage of:}

- Convenient online submission

- Thorough peer review

- No space constraints or color figure charges

- Immediate publication on acceptance

- Inclusion in PubMed, CAS, Scopus and Google Scholar

- Research which is freely available for redistribution 